\title{
Urtication (flogging with stinging nettles) and flagellation (beating with rods) in the treatment of paralysis
}

\author{
J. R. Silver ${ }^{1} \cdot$ M-F. Weiner ${ }^{2}$
}

Received: 3 June 2019 / Revised: 12 August 2019 / Accepted: 23 August 2019

(c) International Spinal Cord Society 2019

\begin{abstract}
Urtication and flagellation were used as a last resort in the treatment of paralysis when all other means were exhausted, and very few cases are reported in the literature. Two cases were identified and reviewed, one of urtication (flogging with nettles) and one of flagellation (beating with rods). In both cases the symptoms were alleviated, but there was insufficient detail to evaluate the therapeutic value of each treatment.
\end{abstract}

\section{Introduction}

The Scottish physician William Buchan (1729-1805), discussed the treatment of palsy in his domestic medicine textbook of 1779. He described how 'Some pretend to have found great benefit from rubbing the affected parts with nettles' [1]. An earlier account by John Locke (1632-1704) in the 17th century referred to the cure of a young woman with palsy after her mother was ordered to 'whip her soundly with rods' [2]. These isolated accounts describe ancient treatments known as 'urtication' or rubbing or flogging with stinging nettles and flagellation.

Paralysis as a result of a fracture or a dislocation of the spinal column has been known for thousands of years but the term 'paraplegia' was not used until the end of the 19th century. The patients were described as suffering from weakness in the limbs, palsy or loss of feeling. Unfortunately, until the 20th century, most patients did not live long enough to have surgery, having succumbed to overwhelming sepsis of the urinary tract and pressure sores in the first few days or weeks after injury. It is therefore both bizarre and surprising that urtication and flagellation should have been used to treat the few surviving patients. This prompted the authors to carry out a review of the 16th to

\footnotetext{
$\triangle$ J. R. Silver

jrussellsilver@btconnect.com

1 National Spinal Injuries Centre, Stoke Mandeville Hospital, Aylesbury, UK

2 Oxford Brookes University, Oxford, UK
}

19th century literature on alternative treatments for various forms of palsy and compare and contrast the use of urtication with nettles and flagellation with rods using two case studies.

\section{Literature review}

The authors concentrated on the British literature from the 16th to the 19th century with anecdotal reference to the French literature. Search words included 'paraplegia, paralysis, palsy, loss of power and muscle weakness for the symptoms/condition and stinging nettles, nettles, urtication, rods, flogging and flagellation for the methods of treatment. Accounts were sought regardless of whether the practitioners were qualified or unqualified, orthodox or unorthodox. The late historian Roy Porter (1946-2002) demonstrated how difficult the distinction can be between these entities and how their fields of practice overlapped, as did their patients [3]. Consequently, established medical journals such as the British Medical Journal, the Lancet and other contemporary textbooks were consulted as was the domestic medicine literature. Finally, the authors deliberately excluded from their study other forms of treatment applied to cases of paralysis (electricity, water therapy, baths, bleeding/bloodletting, the administration of tonics, opiates, purgatives and laxatives) as these are previously documented [4-7].

Whilst stinging nettles have been used for medicinal purposes since antiquity by the Greek physicians Galen (ca. 130-200 AD) and Dioscorides (first century AD), the earliest reference to its use for the treatment of paralysis is by Pliny the Elder (ca. 23-79 AD), a Roman naturalist, who considered it an effective remedy against muscle paralysis (see Fig. 1) [8]. 
Fig. 1 Engraving magnified view of a stinging nettle leaf. From Micrographia: or some physiological descriptions of minute bodies made by magnifying glasses with observations and inquiries thereupon' by Robert Hooke 1665. Reproduced by kind permission of the Wellcome Library London

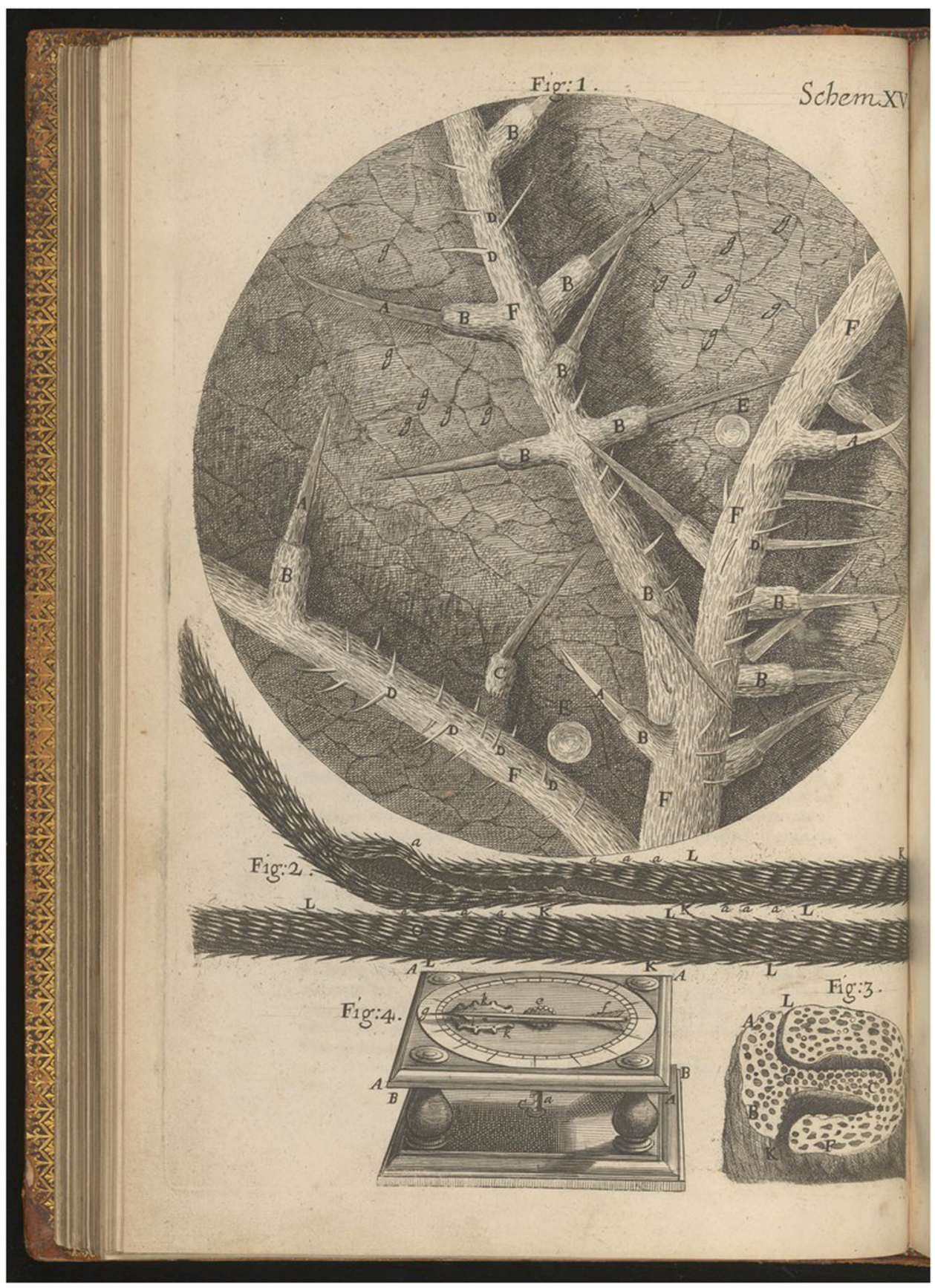

John Piper, an assistant to a surgeon in Birmingham, reports in 1821 on a case of partial paralysis cured by urtication [9].

\section{Account of John Piper}

Spontaneous total paralysis of the right arm for 5 months with loss of feeling and motion [9]. Splinting of the hand with bandage. No previous injury. Pain, oedematous swelling of hands and fingers and contracted tendons. General wasting and flaccidity of the muscular fabric. Whole arm cold and insensitive. Various physicians had previously tried fomentations, frictions, bleedings, electricity, galvanism to no avail.

'She applied successively to six or seven eminent medical gentlemen, who had recourse to a multiplicity of means calculated to restore the suspended energy: amongst which were fomentations, frictions, and a variety of stimulating applications to the limb and along the course of the vertebral column: topical 


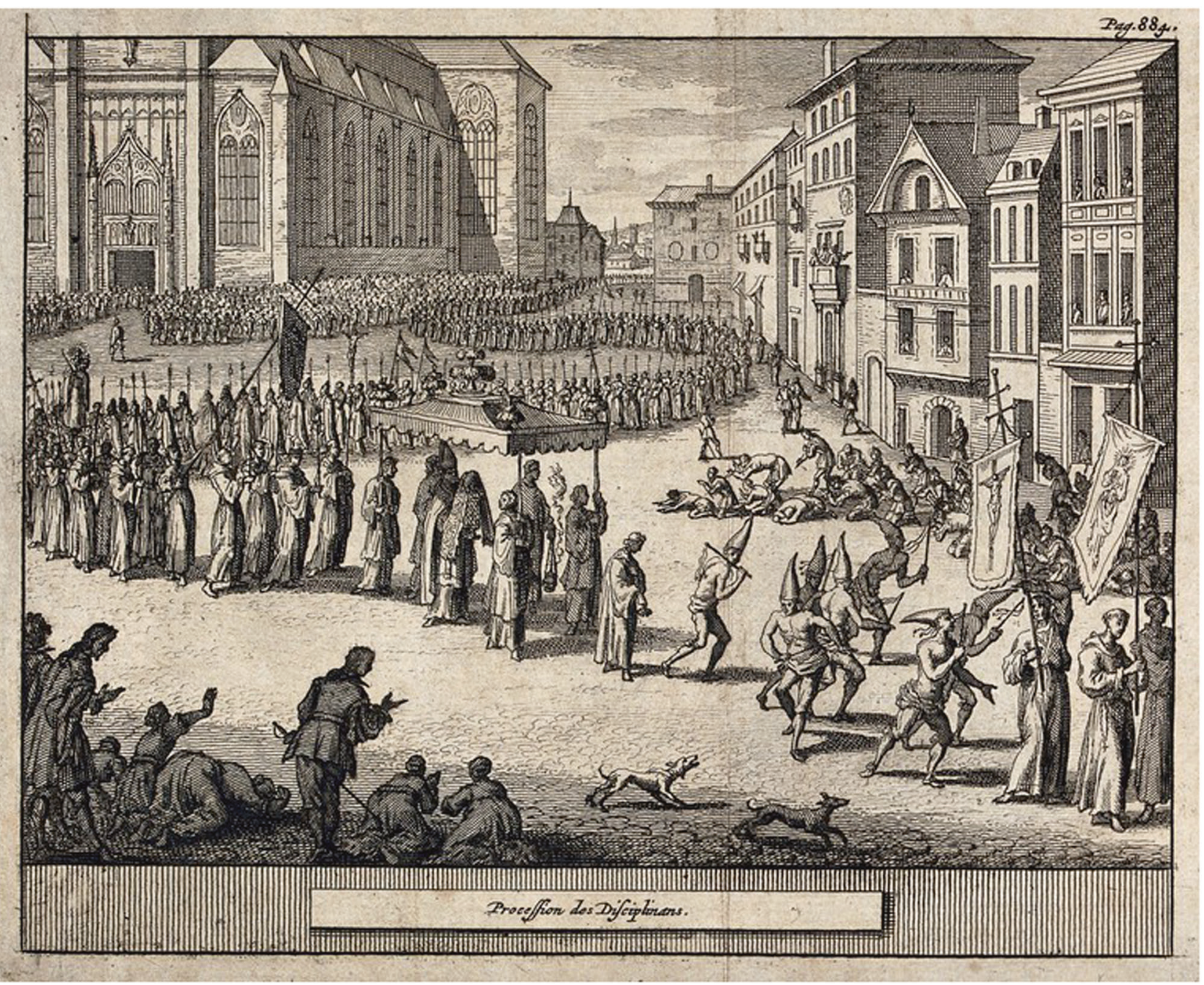

Fig. 2 Flagellants. Reproduced by kind permission of the Wellcome Library London

bleedings by leeches and cupping: vesicatories, electricity, galvanism etc etc.'

Piper used urtication 'a proposition which I perceived excited a risible propensity', indicating the method was widely used. With a rod of nettles he repeatedly inflicted 'piquant flagellation and continued throughout the day'. 'Each application evincing an increased development of sensibility' eventually, 'the long dormant faculties of sensation and motion were perfectly developed; no other trace of the affection remained but a slight weakness of wrist which was soon removed by a strengthening plaster and bandage.'

The practice of flagellation (whipping yourself or someone else, as a religious practice, for punishment, or for sexual pleasure) dates from the earliest ages of mankind but came to prominence in Western Europe in medieval times (see Fig. 2) [10].

Some physicians, however, believed that flagellation had a mechanical basis and reanimated the capillaries, increased muscular energy and promoted the absorption of the secretions of nature. Others looked upon it as another form of bloodletting. John Locke described how Van Helmont cured a case of palsy by beating the patient with rods in the 17 th century.

\section{Account described by John Locke}

'Van Helmont did cure a maid in Rotterdam who by a palsy had lost the use of all her parts from the hips downwards by ordering her Mother to whip her soundly with rods. After whipping, which fetched bloud (original spelling) it washed it with brandy.'

Locke commented that 'perhaps nettles would in the like case be yet better' [11]. Van Helmont (1580-1644), who qualified in medicine in 1599, was an itinerant Flemish 
physiologist and physician who later focused on chemical experiments.

\section{Discussion}

Only two cases are available on which to base our assessment and the details are very limited. Buchan's description refers to the use of stinging nettles but does not provide sufficient detail for an identifiable case. Two distinct forms of treatment for paralysis have been described, urtication and flagellation. At the time the practitioners believed that stinging nettles (urtication) caused a 'chemical' or 'mechanical' reaction in the body with therapeutic benefits to the patient. In contrast, flagellation was a religion-based practice following the concept that disease was visited upon the person as a punishment through an evil spirit and had to be 'beaten out'. Both of these therapeutic approaches resulted in an improvement in the symptoms of paralysis.

With patients suffering from paralysis, it is difficult to assess the therapeutic outcome as the aetiology of the paralysis was unknown and ill-defined. No distinction was made between complete or incomplete loss of power in the lower limbs, traumatic or chronic paralysis or paralysis due to tumours of the spinal cord or secondary deposits from a primary cancer elsewhere, hysterical paralysis, or Pott's disease. The last two categories could recover spontaneously making any evaluation of treatment impossible. Also, post mortems were few and diagnosis was difficult without the modern imaging technology available today (Xray, MRI). The only cases where a clear outcome is known are cases of traumatic paralysis with a complete transection of the cord and these were rare.

\section{Could the treatments have worked?}

The victims of incurable diseases would seek remedies from quacks, charlatans or anyone who could offer them hope. They could fall prey to any kind of magic out of desperation especially if the procedure was dramatic and in an emotional context. It is significant that quacks such as Franz Mesmer (1734-1815) (of mesmerism/hypnotism fame) and James Graham (1745-1794) (of celestial bed fame) exhibited their therapy with dramatic effect. They assailed the senses with music, dramatic costumes and scenery and in some cases sexual overtones. The use of flagellation would amply fulfil these criteria.

Possible benefits of the treatments could have been counter irritation (anti-inflammatory), stimulating of the skin and placebo effect (suggestion). The concept of counter irritation is complex. A pain in one part of the body could be masked by pain applied to an adjacent part such as with a painful cut finger the pain could be alleviated by rubbing the finger or pain from a visceral organ could be masked by a mustard poultice applied to the skin. This theoretical basis of treatment was fashionable in the past with the most recent authoritative endorsement dating of 1890 by Byrom Bramwell in his textbook who refers to counter irritation for the treatment of spinal paralysis (locomotor ataxia) but it has fallen out of fashion. Current textbooks of neurology and medicine make no mention of it [12].

The manifestations and the significance of pain throughout history pose great difficulty. Apart from the intractability of certain forms of pain and one's inability to relieve neurogenic pain, historically pain has had religious connotations. There is an intrusion of religion into medicine. In Babylonian and Egyptian medicine, Jewish Medicine and Christianity, many of the priests were doctors. The injunction in the Bible 'In pain shall you bring forth children' led to objections when effective relief in the form of anaesthesia became available. 'The arguments used by clergy against anaesthesia varied but all centered around the theme of pain, particularly the pain of childbirth...to prevent it was a sacrilege' [13]. Even when general anaesthesia became available for surgery there was reluctance among surgeons to use it as they believed in the therapeutic value of pain $[14,15]$. The situation changed when Queen Victoria, who had many children, was given chloroform during labour.

\section{Contemporary use of urtication and flagellation for medicinal purposes}

The topical application of fresh nettles as a counter irritant for patients with arthritis has a long history and herbal medicine practitioners recommend it still for lower back pain, chronic tendinitis and sprains. Other indications include pain relief in rheumatic complaints and as an adjunct in the treatment of rheumatoid arthritis. However, whilst the plant is recognised as having anti-inflammatory properties, to this date the exact mode of action of the external application of nettles to the skin is unknown and scientific investigations are lacking. The only report on the use of urtication for musculoskeletal pain is by Alford L. (2000), a physiotherapist at the University of East Anglia, who described an isolated case but also reported on a double blind cross-over trial involving 27 patients of whom 17 would elect to use the stinging nettle in the future $[16,17]$. Alford freely conceded that the neuro-physiology underlying the reduction of symptoms is not fully understood although the hypothesis is of a centrally mediated process. The suggestion being that inflicting lower pain can act as a 
'counter-irritant' analgesia, break down scar tissue or reduce a luxation as with many of the methods used today, including acupuncture, sports massages, heat, cold and electric treatment offer a similar proposition.

Medically, the outcome of flagellation could have been equated to another form of blistering or means of fomentation, both of which were advocated in cases of paralysis or general weakness and loss of sensation. Buchan recommended that 'the palsy must be treated in the same way as sanguine apoplexy. The patient must be bled, blistered and have his body opened by sharp clysters or purgative medicines... friction with a flesh brush or a warm hand is extremely proper, especially on the parts affected' [1]. Bleeding was a staple part of treatment from the 16th century and right up to the 20th century. Flagellation is still performed in many countries in the world mostly as a means of punishment and subversion.

Despite evidence that urtication or flagellation with stinging nettles was used by qualified/established practitioners such Culpeper, Buchan and Piper in England and Corvisar in France, accounts of stinging nettles being used in the treatment of paralysis are few [18, 19]. As most patients with a spinal injury died, unusual remedies such as urtication were mostly used to alleviate symptoms in patients with established paralysis as a result of a chronic condition. It could also have been applied to the few surviving patients with spinal injuries as exemplified by Buchan and Locke's (Van Helmont) cases. Unfortunately, there is insufficient detail on the circumstances of the Jan Baptista Van Helmont's patient case as it is a second hand account by John Locke.

These unusual methods of treatment were used after all other means of alleviating symptoms had been exhausted as in the case of Dr Piper's patient. In other instances, by virtue of the patients being isolated in rural areas without easy access to medical care, nettles would have been prescribed firstly because of their reputed analgesic and anti-inflammatory (or counter irritant) properties but also their wide availability in the hedgerows and the fields. The therapeutic properties of the humble stinging nettle continue to exercise the scientific community with many recent publications including an MD thesis on the subject. Today patients and practitioners are assailed with miracle cures.

Acknowledgements We would like to thank Dr Allison Graham and Professor W. El Masri for their constructive advice. We are grateful to the Wellcome Library London for permission to use the illustrations.

\section{Compliance with ethical standards}

Conflict of interest The authors declare that they have no conflict of interest.

Publisher's note Springer Nature remains neutral with regard to jurisdictional claims in published maps and institutional affiliations.

\section{References}

1. Buchan W. Domestic medicine or a treatise on the prevention and cure of diseases by regimen and simple medicines. London: Strahan W. And Cadell T; 1779. p. 469.

2. Evans J, Read S. Maladies \& medicine exploring health \& healing 1540-1740. Barnsley: Pen \& Sword Books Ltd; 2017. p. 21.

3. Porter R. Quacks fakers \& charlatans in English medicine. Stroud: Tempus Publishing Ltd; 2000.

4. Weiner MF, Silver JR. Suspension therapy for the treatment of tabes dorsalis. Eur Neurol. 2014;72:163-72.

5. Silver JR, Weiner MF. Nerve-stretching in the 19th century. J Med Biogr. 2016;24:537-45.

6. Silver JR, Weiner MF. Electrical treatment of spinal cord injuries in the 18th and 19th century. J Med Biogr. 2013;21:75-84.

7. Johnston L. Alternative medicine and spinal cord injury-Beyond the banks of the mainstream. New York: Demos Medical Publishing; 2006.

8. Drexel University. Clinical applications of stinging nettle. College of nursing and health professions. 2012. https://drexel.edu/cnhp/ news/current/archive/2012/August/2012-10-01-Stinging-Nettle/. Accessed March 2019.

9. Piper J. Case of partial paralysis, cured by urtication. Lond Med Phys J. 1821;271:278-80.

10. Ayres L. http://news.bbc.co.uk/1/hi/magazine/8375174.stm. Accessed March 2019.

11. Dewhurst K. John Locke (1632-1704), physician and philosopher: a medical biography with an edition of the medical notes of his journals. London: The Wellcome Library; 1963. p. 274.

12. Bramwell B. Diseases of the spinal cord. 3rd edn. Edinburgh: William F Clay; 1895. p. 357.

13. Haggard H. Devils drugs and doctors: the story of the science of healing from medicine man to doctor. London: William Heinemann Medical Books Ltd; 1929.

14. Heynick F. Jews and medicine an epic saga. Hoboken: Ktav Publishing House Inc; 2002. p. 480.

15. Duncum BM. The development of inhalation anaesthesia with special reference to the years 1846-1900. London, New York, Toronto: Oxford University Press; 1947. p. 165.

16. Alford L. Urtication for musculoskeletal pain. Pain Med. 2008;9:963-5.

17. Randal C, et al. Randomised controlled trial of nettle sting for treatment of base-of-thumb pain. J R Soc Med. 2000;93:305-9.

18. Culpeper N. Culpeper's complete herbal and English physician enlarged. Hertfordshire: UK Wordsworth Editions Ltd; 1995.

19. Cooper WM. Flagellation and flagellants: a history of the rod in all countries from the earliest period to the present time. London: William Reeves; 1880. p. 204, 207, 210-212, 482. 